\title{
Morphological and Histological Study of an 'Iliac Venous Ladder’ Associated with Very Short Common Iliac Arteries
}

\author{
Satheesha B. Nayak ${ }^{1}$ Sudarshan Surendran ${ }^{1} \quad$ Venu Madhav Nelluri ${ }^{1}$ Prakashchandra Shetty ${ }^{1}$ \\ ${ }^{1}$ Department of Anatomy, Melaka Manipal Medical College (Manipal \\ Campus), Manipal Academy of Higher Education, Manipal, \\ Karnataka, India \\ Address for correspondence Sudarshan Surendran, MSc, PhD, \\ Department of Anatomy, Melaka Manipal Medical College (Manipal \\ Campus), Madhav Nagar, Manipal 576104, Karnataka, India \\ (e-mail: sudarsshan.s@manipal.edu).
}

J Morphol Sci 2019;36:255-260.

\begin{abstract}
Keywords

- external iliac vein

- internal iliac vein

- histology

- pelvis

- inferior vena cava

- iliac artery

Introduction Communications between iliac veins in the pelvis are reported to be rare occurrences, which are mostly due to developmental abnormalities. The common iliac vein is formed by the joining of the internal and external iliac veins. Here, we present a detailed morphological and histological study of a rare communication found between the internal and external iliac veins, which would prove to be of substantial value to the knowledge of vessels in the pelvis, both for clinicians and surgeons.

Materials and Methods In the present study, we came across a rare communication in the form of a ladder, between the left internal and external iliac veins in a 70-year-old male cadaver. There were two communications (named upper and lower communications) between the external and internal iliac veins, before they joined to form the common iliac vein. On naked eye observation, the lumen of the right common iliac vein appeared to be wider than on the left side. The lengths of these communications and the distance between each of their attachments have been measured and tabulated. The walls of these veins, their microscopic sections and their communication involved in the case were taken. The possible causes for these variations were congenital, owing to the complicated nature of the developmental process involved in the formation of the inferior vena cava and the venous system of the lower limb.

Results The structure of all the veins involved in this case and the communications were seen to be normal. The thickness of these walls were measured and tabulated for uniformity around the circumference of the wall of these veins. All the measurements from the structural variations and from the histological observations are tabulated in our results.

Conclusion The complicated development sequence of these veins could have possibly led to the persistence of these communications. Such a developmental variation does not seem to pose any threat until unless encountered under clinical or surgical interventions, as the histological structure of the walls of the communications as well as the major channels connected appeared to be normal and well-developed. The detailed morphological and histological features of these structures involved in the variation along with the possible complications have been presented in the present report. Knowledge of these variations and complications due to injury plays a key role in a clinical setup.
\end{abstract}

received

September 5, 2018

accepted

July 20, 2019
DOI https://doi.org/

10.1055/s-0039-1697009.

ISSN 2177-0298.
Copyright $(2019$ by Thieme Revinter

Publicações Ltda, Rio de Janeiro, Brazil
License terms

(1) (1) $\Theta \circledast$ 


\section{Introduction}

The internal and external iliac veins join to form the common iliac vein, where the internal iliac vein drains the pelvic region and the external iliac vein is a continuation of the femoral vein, which drains the lower limb. These two veins join to form the common iliac vein at the pelvic brim, in front of the lower part of the sacroiliac joint. ${ }^{10}$ The developmental formation of the inferior vena cava (IVC) involves a complicated process by the involvement of posterior cardinal, supracardinal, subcardinal, subcardinal and hepatocardiac anastomosis and the hepatocardiac channels. The caudal ends of the posterior cardinal veins receive the veins draining from the pelvis and from the lower limb bud. The involvement of these veins in the formation of the IVC and its tributaries, the multiple connections between these veins, could possibly lead to various anomalous connections between them, if those developmental connections persist. The remnants and its connections (if any) demand a need as it is related to clinical importance and a detailed report regarding these would facilitate clinicians and surgeons to plan their interventions accordingly.

A catheter generally targeted to pass through the IVC is passed through the femoral vein, the external iliac vein and the common iliac vein to reach the IVC. Any variation in this course could lead to life-threatening complications. Variations in the venous system are reported frequently, but the importance is with respect to the vein presenting a critical variation possibly hindering a normal surgical or clinical procedure by a clinician.

Any variation in the normal anatomy of the venous system in this region is related to the developmental process of the retroperitoneal venous system. It is a complex process that consists of a network of venous systems appearing during the initial growth and disappearing at a later stage of the intrauterine life, and this complexity could explain the variations seen in these veins. The subcardinal, the postcardinal and supracardinal veins are seen in pairs on either sides of the midline, and the modifications seen in these sets of veins result in the completion of the development of the retroperitoneal venous system. These developmental changes generally happen between the $6^{\text {th }}$ and $8^{\text {th }}$ weeks of gestation. ${ }^{1}$

In retroperitoneal surgical interventions, such as hysterectomy, the relationship of structures with those considered for surgery play a very important role. Even in imaging of the pelvis, such as a computed tomography (CT) or magnetic resonance imaging (MRI), knowledge of these variations could possibly reduce the risk of assessment that could result in complications. ${ }^{2}$ The internal iliac vein may form a venous annulus, which passes around the internal iliac artery. ${ }^{1}$

In cases of obstruction of the iliocaval segments, the internal iliac vein and its tributaries provide the vital collateral pathways. This factor along with many clinical relevance, adds up to the importance for having a normal development pattern in this venous system. ${ }^{3}$ Any complication in the normal anatomy of this venous system could pose potential problems to clinicians dealing with the pelvis and the structures within the pelvis.

In the present study, we present the involvement of the internal iliac, external iliac and common iliac veins in a variant formation. This variation in the normal anatomy of the veins is of considerable importance especially in surgical or radiological procedures involving the structures present in the pelvic cavity or in the pelvis itself. The aim of the present study was to note the morphological changes of the vessels by measuring them and to check the histological features of their walls.

\section{Materials and Methods}

During our dissection classes for undergraduate medical students, we observed a unique variation of iliac vessels in an adult male cadaver aged $\sim 70$ years old. The overall picture of the vessels of the posterior abdominal wall in relation to the variation is presented here.

The dissection was performed to expose the vessels of the posterior abdominal wall and the pelvis. The terminal branches of the abdominal aorta were traced until their termination. The tributaries of the inferior vena cava from their point of origin were exposed. Relevant photographs of the variations were taken.

The lengths of these vessels were taken using a thread and measured against a normal measuring scale. The values on the scale were noted down (accuracy of $1 \mathrm{~mm}$ ) and the recordings involved two persons to avoid any bias. These values were tabulated for both the right and the left sides. After careful measurement, portions of these variant formations along with the major veins were taken and processed for normal hematoxylin and eosin staining procedures. These tissues were fixed with paraffin wax, then sections were taken using a Leica RM2245 microtome (Leica Microsystems, Wetzlar, Germany). They were mounted on slides, stained with hematoxylin and eosin. The coverslips were fixed using dibutylphthalate polystyrene xylene (DPX).

The wall thickness in these stained sections were measured using an ocular micrometer (Erma Inc., Tokyo, Japan). The values were taken at five different random points that were approximately equidistant from each other. The average of these measurements was tabulated.

\section{Results}

\section{Morphological Observations}

The right and left common iliac arteries were very short, originating from the abdominal aorta at the level of the $4^{\text {th }}$ lumbar vertebra and were $1.0 \mathrm{~cm}$ and $1.5 \mathrm{~cm}$ in length, respectively. This short length of the common iliac arteries led to a higher origin of the internal and external iliac arteries. Their origins were well above the sacral promontory, resulting in increased lengths.

The formation and tributaries of the IVC were normal. On the right side, the external and internal iliac veins had no variations in their normal anatomy. But, on the left side, an 


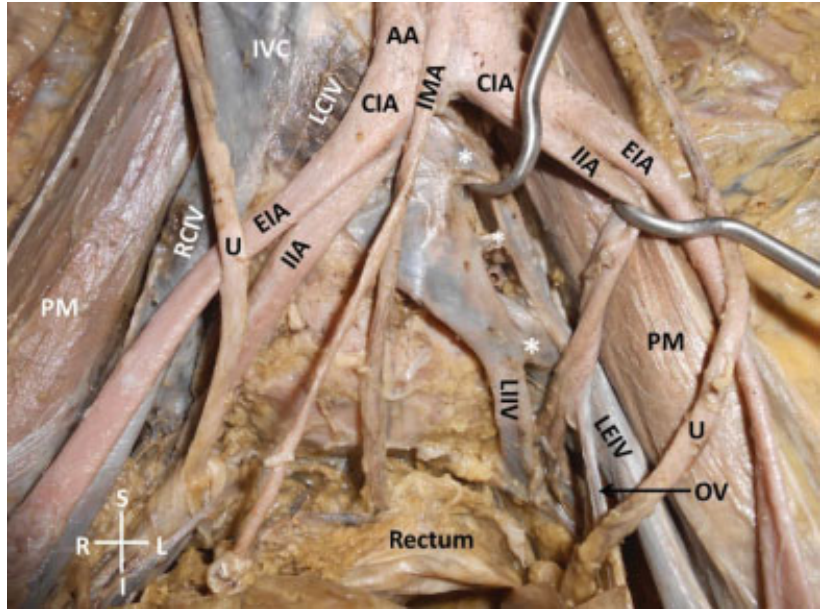

Fig. 1 Dissection of the lower abdomen and pelvis showing the variations of iliac vessels. The asterisks show three levels of opening of the external iliac vein into the internal iliac vein. Abbreviations: $\mathrm{AA}$, abdominal aorta; CIA, common iliac artery; EIA, external iliac artery; I, inferior; IIA, internal iliac artery; IMA, inferior mesenteric artery; IVC, inferior vena cava; L, left; LCIV, left common iliac vein; LEIV, left external iliac vein; LIIV, left internal iliac vein; OV, obturator vein; PM, psoas major muscle; R, right; RCIV, right common iliac vein; S, superior; U, ureter)

enlargement that could be clearly made out by naked eye observation of the common iliac vein was noticed.

In contrast, the left internal iliac vein was observed to be narrower than the right counterpart. The external iliac vein received the obturator, iliolumbar, and superior gluteal veins. There was also an additional large vein from the psoas major muscle draining into it. These tributaries, except for the obturator vein, crossed the obturator nerve and the lumbosacral trunk before they opened into the external iliac vein.

There were two communications seen between the external and internal iliac veins. This venous ladder-like pattern was located above the level of the sacral promontory. It was located lateral to the midline and medial to the psoas major muscle.

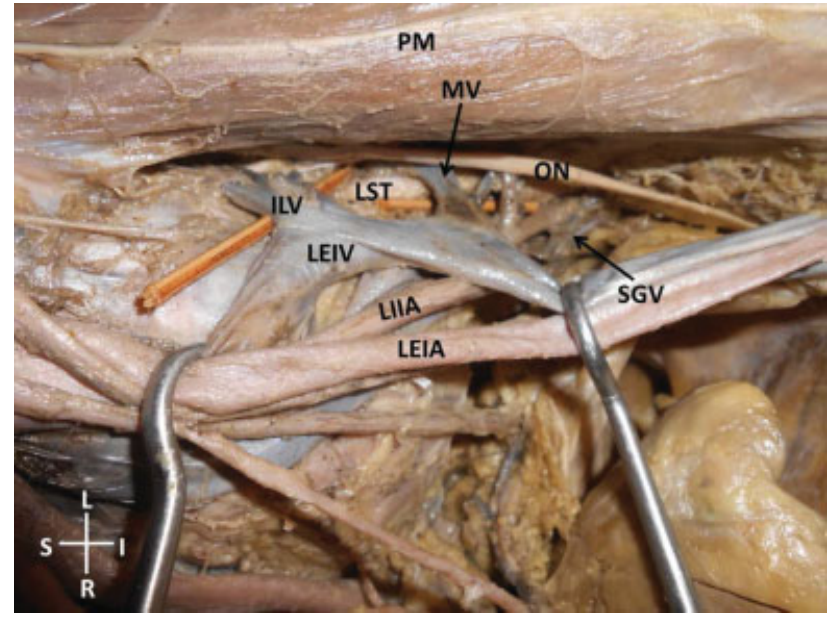

Fig. 2 Dissection of the left wall of the pelvis showing unusual tributaries of the left external iliac vein. Abbreviations: I, inferior; ILV, iliolumbar vein; L, left; LEIA, left external iliac artery; LEIV, left external iliac vein; LIIA, left internal iliac artery; LST, lumbosacral trunk; MV, muscular vein from psoas major; ON, obturator nerve; OV, obturator vein; PM, psoas major muscle; R, right; S, superior; SGV, superior gluteal vein.

These aforementioned variations are shown in -Figs. 1 and 2. The measurements of these veins and the communicating channels between the internal and external iliac veins are tabulated in - Table 1.

\section{Histological Observations}

The measurements were considered in two groups. The first set of values from the normal common iliac veins, external iliac veins, and then the internal iliac veins. Then the measurements were taken from the additional communications between the latter two veins. General microscopic observation revealed the structure of these veins and the communications to be normal with all the three layers of the wall properly formed, owing to their normal developmental

Table 1 Table showing the measurements (length) and extents of the left and right external iliac veins, the upper and lower communicating channels, and the widths of the left and right common iliac veins close to the point of formation and termination

\begin{tabular}{|l|l|l|}
\hline Vein & Extent & Measurement (cm) \\
\hline Left - external iliac vein & From LMIP to LC & 11.0 \\
\hline Left - external iliac vein & Between LC and UC & 2.5 \\
\hline Left - external iliac vein & Between UC and LCIV & 1.5 \\
\hline Right - external iliac vein & From RMIP to RCIV & 15.0 \\
\hline Left - lower communication & From LEIV to LIIV & 1.0 \\
\hline Left - upper communication & From LEIV to LIIV & 0.8 \\
\hline Left - common iliac vein & Width - close to formation & 2.6 \\
\hline Right - common iliac vein & Width - close to formation & 1.2 \\
\hline Left - common iliac vein & Width - close to termination & 2.9 \\
\hline Right - common iliac vein & Width - close to termination & 1.5 \\
\hline
\end{tabular}

Abbreviations: LC, Lower communicating channel; LCIV, Left common iliac vein; LEIV, Left external iliac vein; LIIV, Left internal iliac vein; LMIP, Left mid inguinal point; RCIV, Right common iliac vein; RMIP, Right mid inguinal point; UC, Upper communicating channel. 
Table 2 Thickness of the wall $(\mu)$ of the veins at different points and their mean with standard deviation

\begin{tabular}{|l|l|l|l|l|}
\hline Serial Number & Lower communication & Upper communication & External iliac vein - left & External iliac vein - right \\
\hline 1 & 0.2 & 0.2 & 0.4 & 0.4 \\
\hline 2 & 0.2 & 0.1 & 0.4 & 0.5 \\
\hline 3 & 0.2 & 0.1 & 0.4 & 0.5 \\
\hline 4 & 0.3 & 0.2 & 0.5 & 0.4 \\
\hline 5 & 0.3 & 0.1 & 0.5 & 0.5 \\
\hline Mean \pm SD & $0.24 \pm 0.055$ & $0.14 \pm 0.055$ & $0.44 \pm 0.055$ & $0.46 \pm 0.055$ \\
\hline
\end{tabular}

Abbreviations: SD, standard deviation.

process. The values of their wall thickness at five random points of each of the veins so as to cover the complete circumference are tabulated in -Table $\mathbf{2}$. The histological features are shown in $\boldsymbol{- F i g s . 3 - 6 .}$

\section{Discussion}

The area located between the bifurcation of the aorta above to the pelvic floor below, with internal iliac vessels on the lateral sides, is known as the presacral area. The vessels located in this region are prone to high degrees of variation in this location and there are also reports regarding the variations in the course of the internal iliac vein in this region. ${ }^{4,5}$

According to a previous classification done on internal iliac veins using multidetector CT by Morita et $\mathrm{al}^{6}{ }^{6}$ the variations in the formation of the iliac veins were grouped under seven types, which included dilated Middle Sacral Vein (MSV); on the left - internal iliac vein connecting with the external iliac vein; on the left - the internal iliac vein with an isolated trunk terminating into the common iliac vein; a trunk from the right internal iliac vein terminating into the left common iliac vein; on the right - a trunk separate from the internal iliac vein draining into common iliac vein; on the right - internal iliac vein joining the central common iliac

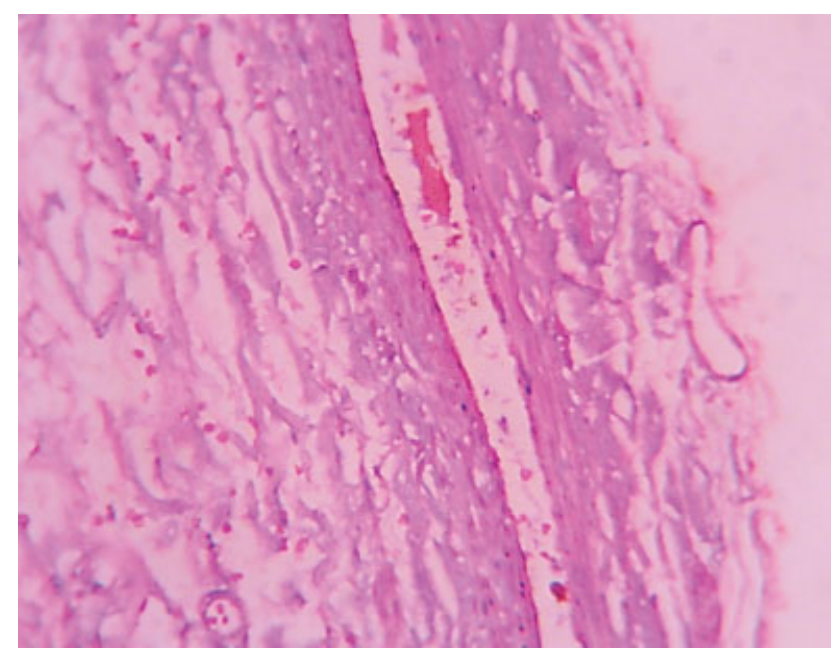

Fig. 3 Photomicrograph showing the microscopic structure of the upper communication channel between the left external iliac vein and the left internal iliac vein. vein; on both sides - additional trunks of internal iliac veins joining with each other and draining into the left central common iliac vein. These are some of the classifications of the iliac vein variations found in the literature. Many of the reports have compared and found the variation under one of these subdivisions. In our case, the multiple connections found in the veins were not found in our literature search.

The understanding of the development of the venous channels in this region could provide an insight into the

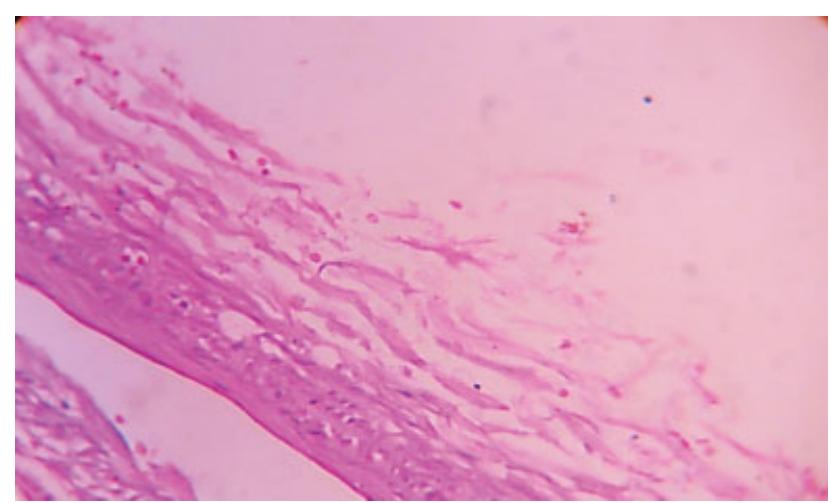

Fig. 4 Photomicrograph showing the microscopic structure of the lower communication channel between the left external iliac vein and the left internal iliac vein.

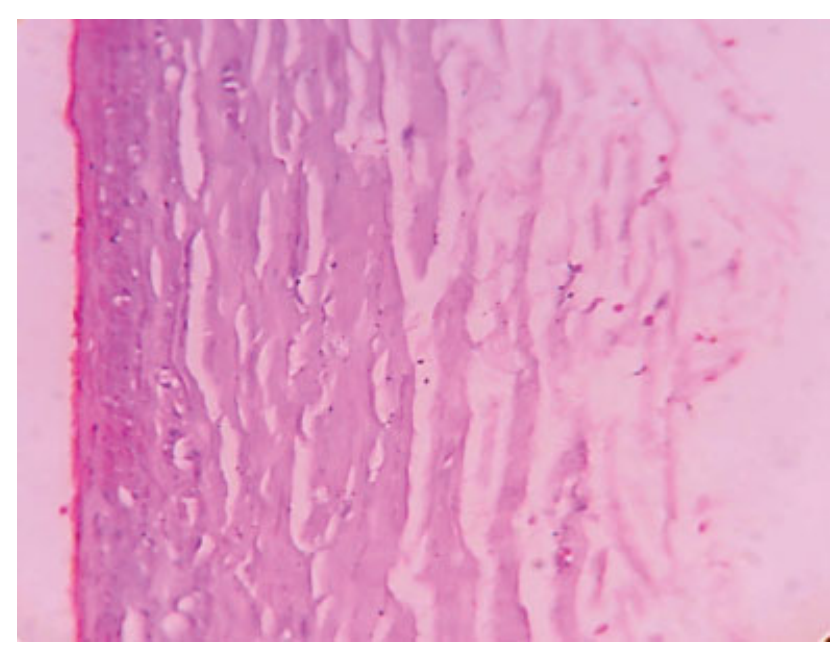

Fig. 5 Photomicrograph showing the microscopic structure of the external iliac vein distal to the communicating channels (both upper and lower) between the left external and left internal iliac veins. 


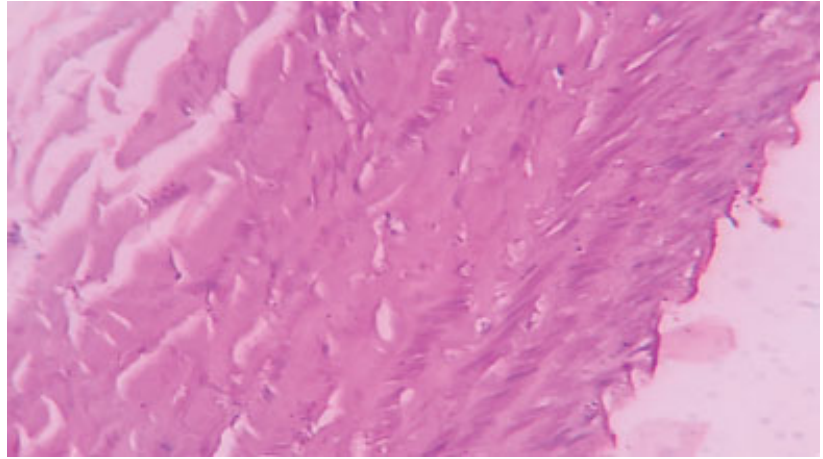

Fig. 6 Photomicrograph showing the microscopic structure of the left common iliac vein.

formation of these variations. The lower limb buds drain into the postcardinal veins, which carry out the function of the inferior vena cava. The definitive left common iliac vein is formed by the oblique transverse anastomosis between the iliac veins formed during development. This anastomosis diverts a huge volume of blood into the right longitudinal veins, which finally account for the disappearance of most of those veins on the left. Finally, the iliac veins and its bifurcations remain as one of the derivatives of the postcardinal veins. $^{7,8}$

This complicated venous channel anastomosis, appearance and disappearance of veins, with many venous channels combining to form a single IVC and veins from both sides joining into this IVC presents a complicated developmental perspective for the veins in this region. A slight variation in the disappearance of a venous channel could be a possible reason for the persistence of these communications between the major veins of the pelvis that drain into the IVC. Although these anomalies generally do not pose a threat to normal functioning of the system and could be attributed to the abnormal developmental process, the complications arising in a clinical or in a surgical environment could not be overlooked. Hence, a detailed knowledge of their presence and their precise location plays a vital role.

Among the complications involved with the venous channels of the pelvic region, sacral colpopexy or an extended lymphadenectomy have been reported to be related to massive bleeding. Complicated vascular structures in this region have been reported in the literature, ${ }^{9,10}$ and there exists a possible life-threatening situation due to damage to any of these complicated vasculatures in this region. ${ }^{11}$ It is also said that a careful dissection to expose the anterior longitudinal ligament of the sacrum prior to the placement of a suture could minimize the complications of these vascularities. Laparoscopic injuries to the retroperitoneal vessels have been attributed not only to the insufficient experience but also to the insufficient knowledge of the anatomy of internal iliac veins. ${ }^{12}$ An overall estimate of the prevalence of structural variations of the internal iliac veins is said to be of $\sim 30.2 \%$, and a surgeon dealing with the pelvic region should be aware of this increased prevalence of variations. In the pathophysiology of the Klippel and Trénaunay Syndrome, there is a surgery requiring the intervention of the internal iliac vein, and in such surgeries, ${ }^{13}$ knowledge of any complicated variations like the ones presented here could prove beneficial for the surgeons. It is also reported that, when placing acetabular screws, the anterosuperior quadrant of the acetabulum should be avoided to prevent injury to the tributaries of the internal iliac vein. A medially placed screw could either penetrate the structures in the area or could be in very close proximity to the tributaries of the internal iliac vein.

During procedures involving catheters through the femoral vein, there could be complications involving lacerations of the veins in the procedure. ${ }^{14}$ As a result of these lacerations, there could be massive retroperitoneal hemorrhage. ${ }^{15}$ It is also suggested that a doctor should ask for help from his colleagues if three attempts to do so are a failure, owing to the complications involved in these procedures. ${ }^{16}$ There are various possibilities for complications in these situations, and our report adds to the existing knowledge of factors.

In the current case, there were two communications between the external iliac vein and the internal iliac vein before the formation of the common iliac vein. These two communications appeared to have a narrow lumen. If the tip of the catheter moves into such a communicating channel during catheterization, there would be a probable restriction in passing the catheter and even a slight increased pressure could rupture them.

Histological sections of these communicating channels and the common iliac veins were performed. Considering the thickness of the venous wall, the layers were normal in the communications, as well as in the common iliac vein. If these channels had improperly developed layers, then it could pose even more danger during surgical procedures. Existence of variations as the one presented in such complicated situations could pose a serious threat to the patients. The knowledge about the variations of these venous systems could be of considerable importance in retroperitoneal surgeries, in certain radiological procedures involving intervention through these veins, and even in the diagnosis of cancer in the pelvis.

\section{Conflicts of Interests}

The authors have no conflicts of interests to declare.

\section{References}

1 Bergman R, Thompson S, Afifi A, Saadeh F. Compendium of human anatomic variation. Urban and Schwarzenberg. Inc.Baltimore, MD1988:93

2 Tanagho EA, McAninch JW, Smith M. General urology. McGrawHill,New York2000

3 LePage PA, Villavicencio JL, Gomez ER, Sheridan MN, Rich NM. The valvular anatomy of the iliac venous system and its clinical implications. JVasc Surg 1991;14(05):678-683

4 Cardinot TM, Aragão AH, Babinski MA, Favorito LA. Rare variation in course and affluence of internal iliac vein due to its anatomical and surgical significance. Surg Radiol Anat 2006;28(04):422-425

5 Oto A, Akpinar E, Sürücü HS, Denk CC, Celik HH. Right internal iliac vein joining the left common iliac vein: case report demonstrated by CT angiography. Surg Radiol Anat 2003;25(3-4):339-341 
6 Morita S, Saito N, Mitsuhashi N. Variations in internal iliac veins detected using multidetector computed tomography. Acta Radiol 2007;48(10):1082-1085

7 Standring S, Ellis HHJ, Johnson D, Williams A. Gray's anatomy: the anatomical basis of clinical practice. 40th ed. Elsevier: Churchill Livingstone; 2008

8 Sürücü HS, Erbil KM, Tastan C, Yener N. Anomalous veins of the retroperitoneum: clinical considerations. Surg Radiol Anat 2001; 23(06):443-445

9 Flynn MK, Romero AA, Amundsen CL, Weidner AC. Vascular anatomy of the presacral space: a fresh tissue cadaver dissection. Am J Obstet Gynecol 2005;192(05):1501-1505

10 Wieslander CK, Rahn DD, McIntire DD, et al. Vascular anatomy of the presacral space in unembalmed female cadavers. Am J Obstet Gynecol 2006;195(06):1736-1741
11 Nygaard IE, McCreery R, Brubaker L, et al; Pelvic Floor Disorders Network. Abdominal sacrocolpopexy: a comprehensive review. Obstet Gynecol 2004;104(04):805-823

12 Kurzel RB, Edinger DD Jr. Injury to the great vessels: a hazard of transabdominal endoscopy. South Med J 1983;76(05):656-657

13 Servelle M. Klippel and Trénaunay's syndrome. 768 operated cases. Ann Surg 1985;201(03):365-373

14 Stolic RV, Milojevic SP. Laceration of the iliac vein in a patient with a femoral catheter for hemodialysis. Int J Nephrol 2011; 2011:919858

15 Yadla M, Sainaresh V, Reddy S, Vijayalakshmi B, Lakshmi A, Sivakumar V. Malposition of hemodialysis catheter in left superior intercostal vein. Hemodial Int 2011;15(01):115-116

16 McGee DC, Gould MK. Preventing complications of central venous catheterization. N Engl J Med 2003;348(12):1123-1133 